\title{
International Journal of Cancer and Oncology
}

Mini Review

\section{Electrical Control of Cancer Cell Migration: A Mini Review}

\author{
Kejun Zhang', Ming Chen', Yong $\mathrm{He}^{2}$, Jianxin Jiang ${ }^{3}$, Min $\mathrm{Zhao}^{4}$, Li Li ${ }^{2 *}$
}

${ }^{1}$ Department of Clinical Laboratory, Daping Hospital, Third Military Medical University, Chongqing 400042, China ${ }^{2}$ Department of Respiratory Disease, Daping Hospital, Third Military Medical University, Chongqing 400042, China ${ }^{3}$ State key laboratory of trauma, burns and combined injury, Daping Hospital, Third Military Medical University, Chongqing 400042, China ${ }^{4}$ Department of Dermatology, Institute for Regenerative Cures, University of California at Davis, CA 95817, USA

\section{Received date: September 28, 2014 \\ Accepted date: December 31, 2014 \\ Published date: January 12, 2015}

*Corresponding author: $\mathrm{Li} \mathrm{Li}$, Department of Respiratory Disease, Daping Hospital, Third Military Medical University, Chongqing 400042, China. Tel: 86-23-68757793, E-mail: 1ili10119@126.com

Keywords: Electric fields; Cancer invasion; Directional migration; Metastasis

Citation: Li Li., et al. Electrical Control of Cancer Cell Migration: A Mini Review. (2015) Int J Cancer Oncol 2(1): 1-3.

\section{Introduction}

For multiple types of cancer, the major cause of death is the development of metastatic diseases ${ }^{[1]}$. Metastasis is a multistep process where cancer cells invade surrounding tissues and blood vessels, and establish secondary tumors in distant organs ${ }^{[2]}$. In detail, these steps include local invasion, intravasation, survival in the circulation, extravasations, and colonization. The mechanisms of metastasis are not fully understood. Various environmental factors, including cytokines and growth factors from autocrine and paracrine sources, have been demonstrated to stimulate cancer cell migration by creating chemo tactic signals ${ }^{[3]}$.Aside from those commonly known chemical guidance, the endogenous electric fields (EFs) has also been proved to be an important directional cue for directional cell migration.

\section{Possible endogenous EFs at sites of cancer invasion}

Endogenous EFs was initially discovered more than 150 years ago from a cut in his own finger by Du Bois-Reymond. Polarized epithelia transport ions directionally and maintain trans-epithelial potentials (TEP).The TEPs are present in many types of epithelia, including respiratory, gastrointestinal, urinary, and bile duct systems, and in prostate, breast, cerebral cavities, retina, and ocular lens ${ }^{[4-14]}$. When a wound occurs, it breaks the barrier and instantly short-circuits the TEP, and then generates wound EFs. This wound-induced electrical signal was as large as $42-150 \mathrm{mV} / \mathrm{mm}$, detected by micro needle arrays and Bio-Electric Imager ${ }^{\circledR}{ }^{[15,16]}$. Furthermore, it could last for many hours and regulate different cell behaviors within $500 \mu \mathrm{m}$ to
$1 \mathrm{~mm}$ from the wound edge until re epithelialization occurs ${ }^{[17]}$. During the last decade, EFs has been proved to be an overriding signal than other directional cues in guiding cell migration in wound healing ${ }^{[18]}$.

In most tumor cells, the membrane potential is depolarized, thus causing a voltage drop between the cancerous and normal tissue ${ }^{[19]}$. To become migratory, the first barrier that tumor cells must overcome is their epithelial basement membrane ${ }^{[20]}$. When tumor cells form invadopodia and cross the basement membrane, it is likely a "wound" occurs at the epithelial membrane, thus may generate endogenous EFs. Actually, at the tissue surface of cancerous and normal tissues, potential gradients can be measured and used clinically to diagnose early-onset breast cancer ${ }^{[21]}$. Also, as demonstrated earlier in other studies, epithelial cells in certain areas of the breast cancer divide more rapidly than cells in normal areas, which results in significantly greater electro potential difference on the skin surface over invasive cancer than benign regions ${ }^{[22]}$. The endogenous EFs at sites of cancer invasion might play an important role in directing migration direction of cancer cells.

\section{EFs direct migration of multiple types of cancer cells}

As stated above, cell migration is a key element in tumor progression. Understanding whether naturally occurring or applied electrical signals can control migration of tumor cells might offer novel strategies to prevent cancer metastasis. Indeed, studies in the last decade have demonstrated that EFs guide directional migration of multiple types of cancer cells, including lung can-

Copy rights: (C2015 Li Li. This is an Open access article distributed under the terms of Creative Commons Attribution 4.0 International License. 
cer cells, breast cancer cells, prostate cancer cells, fibro sarcoma cells and so on ${ }^{[22-25]}$. Interestingly, some types of cancer cells migrate to the cathode in an EF, while others to the anode, indicating that electrotaxis of different cancer cells can be characteristically different in specific disease models.

Tumor cell electrotaxis also correlates with metastatic potential. For example, the highly metastatic rat breast cancer cell line MTLn3 showed a robust anodal galvanotactic response, whereas non-metastatic MTC cells responded three times less to an electric field of the same strength ${ }^{[22]}$. For two sub clones of CL1 lung adeno carcinoma cells, the highly invasive CL 1-5 cells are anodally electro tactic while the low invasive CL 1-0 cells are non-electro tactic ${ }^{[23]}$. Similarly, Djamgoz et al have shown different galvanotactic responses of two prostate cancer cell lines. The highly metastatic MAT-lyLU cells migrated directionally to the cathode, while the weakly metastatic AT-2 cells responded poorly to an EF of the same strength ${ }^{[26]}$. Thus, the ability of cancer cells to respond to an EF could potentially reflect its metastasis potential.

EFs may also be an important tool to control collective cancer invasion. Growing evidence indicates that collective cell migration plays a part in cancer invasion. In histo pathological sections of several types of epithelial cancers, the primary tumor is surrounded by secondary cancer cells in the form of clusters, chains or sheets ${ }^{[27]}$. Also, many types of epithelial cancers, breast cancer and colorectal carcinoma, for example, exhibit collective invasion in cultures ${ }^{[27,28]}$. Previously, we have shown that EFs guide directional migration of large epithelial sheets. More importantly, collectively cells respond better to EFs than cells in isolation, which depends on E-cadherin-mediated cell-cell adhe$\operatorname{sion}^{[29]}$. Of note, intact cell-cell junctions as well as the expression of E-cadherins and other adhesion molecules were found in histo pathological sections of multiple types of epithelial cancers $^{[30]}$, thus indicating that EFs may guide collective invasion of cancer cells.

How electric fields guide directional migration of cancer cells? Up to now, several mechanisms have been suggested to be involved in electrotaxis of different types of cancer. EFs control directional migration of prostate cancer cells through modulation of voltage-gated sodium channels ${ }^{[31]}$. Pu et al reported that electric signals enhanced breast cancer cell migration through the ErbB-signalling pathway ${ }^{[22]}$. Still, activation of ERK and reorganization of the cytoskeleton play a part in electrotaxis of fibrosarcoma cells ${ }^{[25]}$.

\section{Future perspectives}

The importance of electrical control of cancer invasion has aroused increasing attention in recent years, yet there's still a lot that needs to study. Firstly, in vivo models testing the effect of either application of external EFs or modulation of endogenous EFs on cancer invasion are urgently needed. As discussed above, most cancer cell lines tested so far respond to EFs in vitro, yet there's little evidence that they actually follow the direction of EFs to migrate and metastasize in vivo. Secondly, besides controlling migration direction, EFs may affect other aspects of cancer biology. Growth of blood vessels is essential to cancer growth, while EFs have been proved to be a significant directional cue for angiogenesis, and migration of endothelial cells and endothelial progenitor cells ${ }^{[32,33]}$. Thus, EFs may control both the migration direction of cancer cells and the growth direction of local blood vessels. Thirdly, further studies on mechanisms by which EFs control directional cancer invasion are still needed. As stated above, several signaling pathways play a role in directional migration of cancer cells in an EF. Are there any other signaling pathways involved in cancer cell electrotaxis? Do these mechanisms exist in vivo? Could they be potential therapeutic targets to inhibit cancer invasion?

In summary, EFs is increasingly recognized as a significant directional cue that drives directional cancer invasion. Understanding the common rules of electrical control of directional cancer migration will lead to the development of strategies that inhibit or prevent cancer invasion or metastasis.

Source of support: This work was supported by National Nature Science Foundation of China (81172113 and 81301840), a foundation for PLA Young Scientists (13QNP117), and a foundation of Third Military Medical University (2012XLC08).

Conflict of interest: The authors disclose no potential conflicts of interest.

\section{References:}

1. Siegel, R., Ward, E., Brawley, O., et al.Cancer statistics, 2011: the impact of eliminating socioeconomic and racial disparities on premature cancer deaths. (2011) CA Cancer J Clin 61(4): 212-236.

2. Talmadge, J.E., Fidler, I.J. AACR centennial series: the biology of cancer metastasis: historical perspective. (2010) Cancer research 70(14): 5649-5669.

3. Levine, M.D., Liotta, L.A., Stracke, M.L. Stimulation and regulation of tumor cell motility in invasion and metastasis. (1995) EXS 74:157179.

4. Friedman, P.A., Gesek, F.A. Cellular calcium transport in renal epithelia: measurement, mechanisms, and regulation. (1995) Physiol Rev 75(3): 429-471.

5. Kunzelmann, K., Mall, M. Electrolyte transport in the mammalian colon: mechanisms and implications for disease. (2002) Physiol Rev 82(1): 245-289.

6. Larsen, E.H. Chloride transport by high-resistance heterocellular epithelia. (1991) Physiol Rev 71(1): 235-283.

7. Macknight, A.D., DiBona, D.R., Leaf, A. Sodium transport across toad urinary bladder: a model "tight" epithelium. (1980) Physiol Rev 60(3): 615-715.

8. Mathias, R.T., Rae, J.L., Baldo, G.J. Physiological properties of the normal lens. (1997) Physiol Rev 77(1): 21-50.

9. Meyer, G., Guizzardi, F., Rodighiero, S., et al. Ion transport across the gallbladder epithelium. (2005) Curr Drug Targets Immune, Endocr Metabol Disord 5(2):143-151.

10. Turnheim, K. Intrinsic regulation of apical sodium entry in epithelia. (1991) Physiol Rev 71(2): 429-445.

11. Van Driessche, W., Zeiske, W. Ionic channels in epithelial cell membranes. (1985) Physiol Rev 65(4): 833-903.

12. Welsh, M.J. Electrolyte transport by airway epithelia. (1987) Physiol Rev 67(4): 1143-1184.

13. Maminishkis, A., Chen, S., Jalickee, S., et al. Confluent monolayers of cultured human fetal retinal pigment epithelium exhibit morphology and physiology of native tissue. (2006) Invest Ophthalmol Vis Sci 47(8): 3612-3624.

14. Hammerton, R.W., Krzeminski, K.A., Mays, R.W., et al. Mechanism for regulating cell surface distribution of $\mathrm{Na}+, \mathrm{K}(+)$-ATPase in polarized epithelial cells. (1991) Science 254(5033): 847-850.

15. Mukerjee, E.V., Isseroff, R.R., Nuccitelli, R., et al.Microneedle array for measuring wound generated electric fields. (2006) Conf Proc IEEE Eng Med Biol Soc 1: 4326-4328. 
16. Nuccitelli, R., Nuccitelli, P., Ramlatchan, S., et al. Imaging the electric field associated with mouse and human skin wounds. (2008) Wound Repair Regen 16(3): 432-441.

17. McCaig, C.D., Rajnicek, A.M., Song, B., et al. Controlling cell behavior electrically: current views and future potential. (2005) Physiol Rev 85(3): 943-978.

18. Zhao, M. Electrical fields in wound healing-An overriding signal that directs cell migration. (2009) Semin Cell Dev Biol 20(6): 674-682. 19. McCaig, C.D., Song, B., Rajnicek, A.M. Electrical dimensions in cell science. (2009) J Cell Sci 122(23): 4267-4276.

20. Bravo-Cordero, J.J., Hodgson, L., Condeelis, J. Directed cell invasion and migration during metastasis. (2012) Curr Opin Cell Biol 24(2): 277-283.

21. Cuzick, J., Holland, R., Barth, V., et al. Electropotential measurements as a new diagnostic modality for breast cancer. (1998) Lancet 352(9125): 359-363.

22. Pu, J., McCaig, C.D., Cao, L., et al. EGF receptor signalling is essential for electric-field-directed migration of breast cancer cells. (2007) J Cell Sci 120(Pt 19): 3395-3403.

23. Tsai, H.F., Huang, C.W., Chang, H.F., et al. Evaluation of EGFR and RTK signaling in the electrotaxis of lung adenocarcinoma cells under direct-current electric field stimulation. (2013) PLoS ONE 8(8): e73418.

24. Martin-Granados, C., Prescott, A.R., Van Dessel, N., et al. A role for PP1/NIPP1 in steering migration of human cancer cells. (2012) PLoS ONE 7(7): e40769.
25. Li, F., Wang, H., Li, L., et al. Superoxide plays critical roles in electrotaxis of fibrosarcoma cells via activation of ERK and reorganization of the cytoskeleton. (2012) Free Radic Biol Med 52(9):1888-1896.

26. Dittmar, T., Husemann, A., Schewe, Y., et al. Induction of cancer cell migration by epidermal growth factor is initiated by specific phosphorylation of tyrosine 1248 of c-erbB-2 receptor via EGFR. (2002) FASEB J 16(13): 1823-1825.

27. Nabeshima, K., Inoue, T., Shimao, Y., et al. Cohort migration of carcinoma cells: differentiated colorectal carcinoma cells move as coherent cell clusters or sheets. (1999) Histol Histopathol 14(4):1183-1197.

28. Friedl, P., Noble, P,B, Walton, P.A., et al. Migration of coordinated cell clusters in mesenchymal and epithelial cancer explants in vitro. (1995) Cancer Res 55(20): 4557-4560.

29. Li, L., Hartley, R., Reiss, B., et al. E-cadherin plays an essential role in collective directional migration of large epithelial sheets. (2012) Cell Mol Life Sci 69(16): 2779-2789.

30. Friedl, P., Gilmour, D. Collective cell migration in morphogenesis, regeneration and cancer. (2009) Nat Rev Mol Cell Biol 10(7): 445-457. 31. Mycielska, M.E., Djamgoz, M.B. Cellular mechanisms of direct-current electric field effects: galvanotaxis and metastatic disease. (2004) J Cell Sci 117(Pt 9): 1631-1619.

32. Zhao, Z., Qin, L., Reid, B., et al. Directing migration of endothelial progenitor cells with applied DC electric fields. (2012) Stem Cell Res 8(1): 38-48.

33. Bai, H., Forrester, J.V., Zhao, M. DC electric stimulation upregulates angiogenic factors in endothelial cells through activation of VEGF receptors. (2011) Cytokine 55(1): 110-11 5. 